University for Business and Technology in Kosovo

UBT Knowledge Center

UBT International Conference

2014 UBT International Conference

Nov 7th, 5:30 PM - 5:45 PM

\title{
On Form and Logic of Structures
}

Feti Selmani

PAN Engineering, panengineering@gmail.com

Follow this and additional works at: https://knowledgecenter.ubt-uni.net/conference

Part of the Architecture Commons

\section{Recommended Citation}

Selmani, Feti, "On Form and Logic of Structures" (2014). UBT International Conference. 8.

https://knowledgecenter.ubt-uni.net/conference/2014/all-events/8

This Event is brought to you for free and open access by the Publication and Journals at UBT Knowledge Center. It has been accepted for inclusion in UBT International Conference by an authorized administrator of UBT Knowledge Center. For more information, please contact knowledge.center@ubt-uni.net. 


\title{
On Form and Logic of Structures
}

\author{
Feti Selmani \\ PAN Engineering, Structurosys, Prishtinë, Kosovo \\ panengineering@gmail.com
}

\begin{abstract}
In Civil Engineering, every structure harbours some degree of logic within it. Sometimes, it is easy to see it, while sometimes it lies somehow hidden and only a well informed eye can take hold of it. The Natural phenomena are dual in their manifestation. So are Tension and Compression, which represents the basic "genomes" of this interaction and are present within each structure from the simplest to the most complicated one. A structural form represents a physical manifestation of this invisible interplay, which, when in consonance with the natural flow of forces results not only in an economic but in a well proportionate and aesthetic structure - a work of art. Nowadays, the culture of putting unreserved belief on the results given by supercomputers slowly degraded in becoming a tradition. This paper calls for an increase to awareness within the community of structural engineers by visiting some of the basic principles on which our profession holds on.
\end{abstract}

Keywords: Structures, logic, compression, physical

\section{Introduction}

Natural phenomena occur in accordance to certain laws. In his well known book "On Growth and Form" [1], D'Arcy Thompson wrote: ("We learn and learn, but never know it all, about the smallest, humblest thing...". Such is the way of learning, that even today, we are not sure if nature creates in a disciplined or in a chaotic and irregular way. Finding the truth remains the holy grail of science. Yet, the truth remains a very special "commodity" in our way of seeking - if not for something else, then just for the sake of being so difficult to find it.

Referring to the civil engineering problems, nowadays one of the greatest challenges remains the imitation of natural phenomena and laws through modelling. Of course, we can tell that the truth has been achieved when obtained results both through mathematical and experimental findings come near. One of natural's phenomena having a great impact on civil engineering structures is the gravity. During the design of an edifice one must never forget its influence. Therefore, when one seeks to be as "original" as possible he mustn't underestimate the natural flow of forces, since the consequences could be catastrophic. Even the horizontal actions such as the seismic forces are a product of gravitational forces; in fact they do represent a certain percentage of them.

Actually, everywhere around the world one can see a strong tendency of designing and building "spectacular" structures - bee it in the plane or in the height. And, without putting any rigid constrains on neither visual expressions nor architectural styles, we can stress that any building designed and built on excess of respecting basic principles such as: simplicity, regularity, symmetry, repetition and similarity through variation of forms [2- fs], cannot result to be in-line with neither resources in disposal nor general aesthetical expectations.

\section{Form and Mechanical Strength of a Structure}

A structural pattern is aimed at offering a global resistance versus external natural phenomena such as wind or earthquake and must fulfil the required level of performance. This global resistance of a 
structure is composed, however, from localy supplied strengths, distributed throughout structural elements.

That a structure's various levels of resistance depends from its form, i.e., the arrangement of its parts is known a thousand years before the science on structures found herself put on modern rails [10 - Karl Eugene Kurrer], that is on fundamental mechanical principles. Before this so called discipline-formation period [10] the structural art was based on a culture established more on the culture of build-ruin-rebuild than a scientific one. It is impressive, however, even though our predecessors didn't posses any knowledge of the above mentioned principles, they achieved creating magnificent works of art, which, even today, astound us with their elegance and beauty. The form and the three-dimensional configuration have a great influence on the strength of a structure towards external disturbances on the way to fulfil both the serviceability and no-collapse requirement. The selection of this form depends of course, from several parameters as well as constrains of different nature such as: cultural and social backgrounds, economic ones, state of know-how ect.
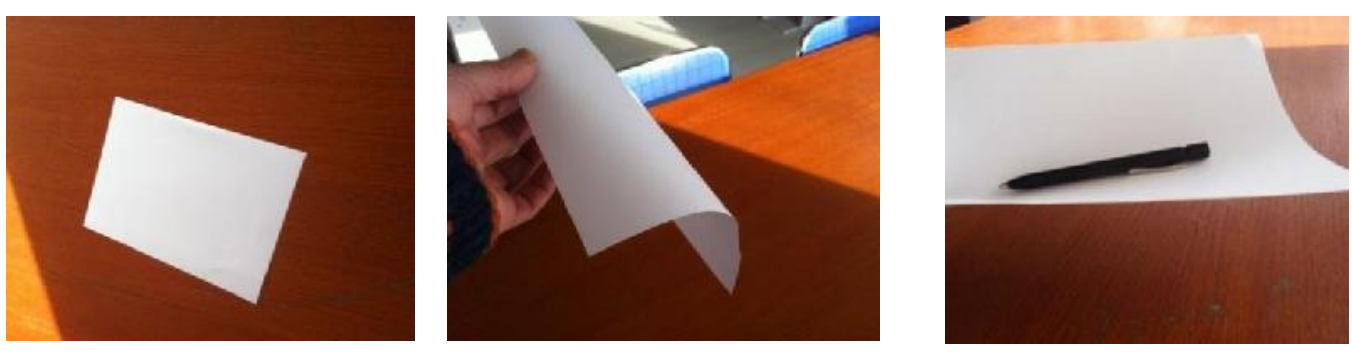

Fig. 1. 1 Left: A4 sheet of paper in plane $(\boldsymbol{\rho}=\mathbf{0})$; in the middle: A4 sheet given a certain curvature $\boldsymbol{\rho}=\boldsymbol{\rho}_{0}$; right: the generated cylindrical surface and the shear increase of the bearing capacity $(\boldsymbol{\rho}=$ $\left.\rho_{0}\right)$.

Once this process in hands of a skilful structural designer, one can produce shapes and forms being in complete harmony not only with natural laws but in harmony with the nature itself and its surroundings. Let take for example, as shown in the Fig.1.1 further below the case of a sheet of paper, which, when given a certain curvature $\rho$ becomes able not only to sustain himself, but is able to support objects exceeding several times his own self-weight.

Where lays the secrecy of the increased bearing capacity of this primitive structure? It exactly lies in its change of shape, i.e., increasing of its stiffness through form alteration in the three-dimensional space. This is achieved through the increase of its curvature from $\rho=0$ (plane) to $\rho=\rho_{0}$ in space. Mathematically, this can be expressed through the expression given as per equation (1.1), whereas physically, this can be explained through the internal increase of bending capacity from a rather inexistent value $M \cong 0$ to a specific value $M=M_{0}$.

$$
M / E I=1 / r=\rho
$$

Important to the mechanical resistance are not only the form and its constitutive materials but the geometry and cross-section of its elements. Biostructures - the structures created by nature are a beautiful example to follow from the part of structural designer as their cross-sectional shape ensues from the evolutionary process of self-adaptation and natural selection [Darwin]. As an example it can be illustrated the wheat steam cross-section (Fig.1.2 right insert). To the question of why this happened, why did the Nature "chose" this cross-section, one can give a very simple answer: during his lifetime the wheat stem is submitted to wind actions coming from various direction and must adapt itself if it wants to survive, therefore there is no better response than tubular cross-section which has the same 

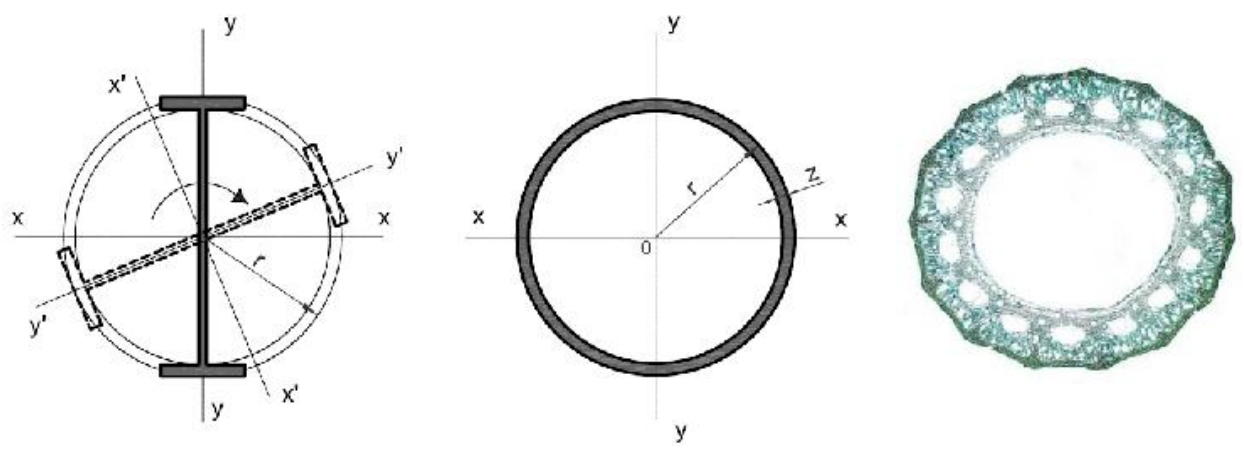

Fig. 1.2

Left: cross-section in form of "I"; middle: the tube; right: wheat stem's cross-section - biostructure.

module of resistance in all directions and a very good resistance towards torsion. Clearly, Nature did the right selection!

It is of a special interest to us structural designers for this adaptation process to be decoded through some kind of comparative anatomy, in order to produce structural shapes that have not only achieved the required strength but in the same time the economy of mass and mater. To illustrate this, let us study one of the most simple and widely used cross-sections - the rectangular one. If we take-out the mass belonging to the proximity of the neutral axis, we obtain the structural shape in the form of $I$ consisting of flanges and the vertical web - widely used in metal structures (Fig.1.2 - left insert). Even though this small "surgery" brings an improvement as far as the stiffness of the cross-section regards, this is not satisfactory because this enhancement is spread through two "principal" axes ( $y-y$ - in our case). In the case we continue further and let the $I$ profile rotate around its longitudinal axis (pole 0 , Fig. 1.2 - middle insert), then the tube results (pole O, Fig.1.2 - right insert). This is exactly what Mother Nature has chosen during the evolution, and the Engineer adopted it intelligently also!

\section{Tension and Compression}

As said before, natural phenomena are of a dual character. There is no better expression than that of a harmony between two phenomena contrary to each other, yet, complementary in order to form a whole. In Mechanics, the internal competition of this duality is manifested by the way of tension and compression. Beginning from the most primitive to the most complex structure, this two phenomena reign supreme.

One of the best descriptions of this relation was given by R. Buckminster Fuller (1895-1983), [RBFuller] as follows: "Compression is inherently partial. Tension is inherently total".

Hereunder there will be given some illustrations in order to show this powerful interaction between the two phenomena, beginning from biostructures to the civil engineering structures. Later on some of the most renowned works in architecture, known not only for their aesthetical values but above all their structural efficiency shall be illustrated, with emphasis to the duality between tension and compression.

There is no a better case in nature of a structure (bio-) working exclusively in tension than dowry's leaf (Fig. 2.1 - in the middle and right insert). On the other side - Fig. 2.1, left insert, the human body when taken as isolated, works exclusively in compression under the action of gravity. This action arises from the universal law of gravitation as given per expression (2.1) below:

$$
F_{1,2}=F_{2,1}=G \cdot \frac{m_{1} \cdot m_{2}}{r^{2}}
$$


By treating the human body as a subsystem, it becomes one of the most perfect mechanisms in the universe (for as much as we know!) - a tensegrity structure [BF], i.e., a mechanism where the tension is continuous and in complete harmony with compression.

In the Fig. 2.2, further below it is shown a dowry's leaf, whilst at the right insert of the same figure one can see the clover blossom (trifolium pratense) submitted to the morning dew' load. From the biology we learned the utility of the leaf, which are the absorption of carbon dioxide and the production of oxygen through the photosynthesis. An important fact, here, it is of how Nature designed the leaf structure: here, one of the most fundamental Nature's principles can be seen - the generation of minimal plane surface. The reason for this is quite evident: Nature wants to expose the leaf cells as much as possible to the sun's rays, in order to absorb as much carbon-dioxide as possible and to produce as much needed oxygen as possible. Beautifully engineered, not!

As far as the right insert on the figure we have one another case of a beautifully crafted structure: the morning dew represents a sphere which is created through surface tension phenomena and represent a minimal area, which is the smallest surface generated versus the given volume - see expression (2.2)

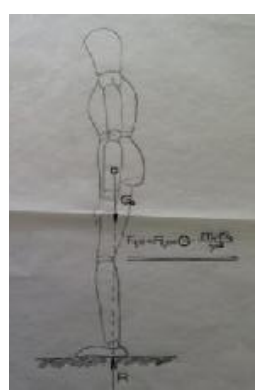

$$
r=S / V=4 \cdot \pi \cdot r^{2} /\left((4 / 3) \cdot \pi \cdot r^{3}\right)=(1 / 3) r
$$
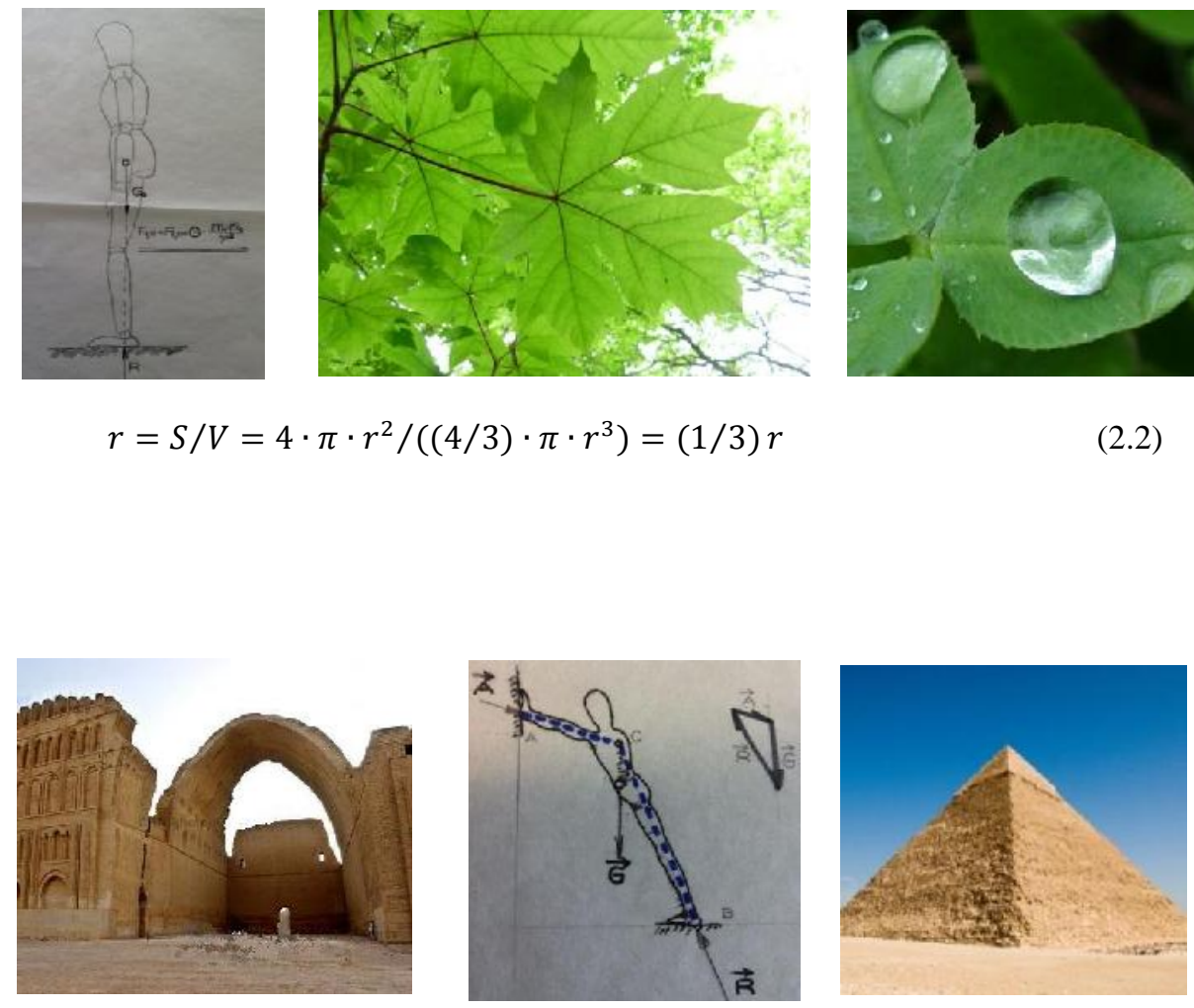

Fig. 2.2 Left: human body and the forces acting on it; middle: dowry's leaf; right: clover blossom (trifolium pratense) submitted to the morning dew' load. 
Fig.2.3 Left: Ctesiphon palace, $1^{\text {st }}$ century BC; middle: man leaning on the wall - analogy; right: Great Pyramid of Giza, 2500 BC.

\subsection{Compression}

From ancient times to the present day, one of the most used structural forms was the vault or in its simplest of forms - the arch. The main characteristic of the vault is that it works mainly in compression. In the Fig. 2.3 below, one can see one of the most utilized cylindrical vaulted structures - mainly executed in antiquity. It is clearly seen that its thickness increases gradually towards the supports in order to compensate the horizontal thrust of the vault, that is, the deviation of the resultant of internal

forces (funicular polygon). This act although highly effective in insuring the necessary vault stability was done empirically and was not based in any technical or scientific knowledge. What is beautiful with this detailing is not just the stability in a pure engineering sense - it is the visual expression of elegance and psychological assurance for the user that the vault is strong and won't fall down.

The Fig. 2.4 below shows the two-hinged arch, which for the case of a uniformly distributed load has a funicular polygon whose form can be mathematically described through a second degree parabola (Fig. 2.4 - a). In the case the uniform load acts on the left-half or the right-half of the arch, then funicular polygon adapts himself the position of external loads and descends or rises-up in order to permit to the arch to compensate the deformations caused by it $(F i g .2 .4-b, c)$.

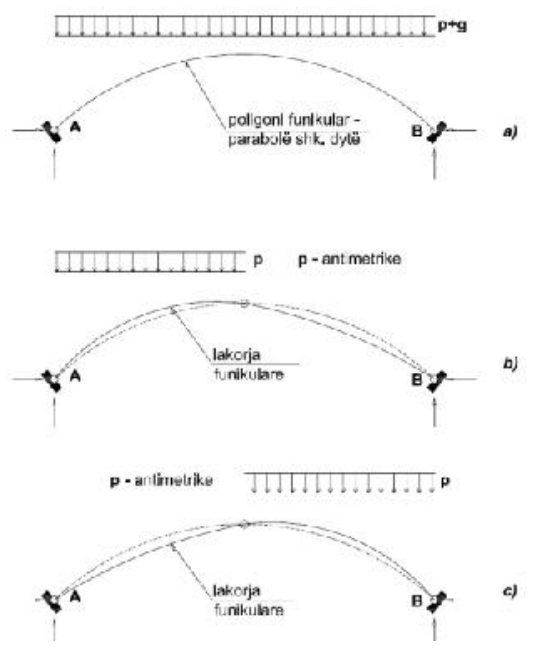

Fig. 2.4 a) Two-hinged arch under the uniformly distributed loads $p+g ; b \& c$ ) two-hinged arch under the action of antimetric load $p$, dashed line - undeformed arch, continuous line - deformed arch. The geometry (the form) of the arch and the mechanical efficiency are closely related between them. Thus, the factors that have the most influence on the cross-sectional dimensions of the arched element are the slenderness ratio $l / f$ as well as the ratio $(p+g) / g$, where: $p$ - is the variable uniform action and $g$ is the sum of all permanent actions acting on the structure (self weight plus super dead loads).

A well known problem being present in arched structures is the loos of stability which is usually solved by introducing the stiffening beam (intrados or extrados) as shown in the Fig. 2.5 below. In the figure one can witness one of the most celebrated cases in the history of theory of structures - the three-arched bridge of Robert Maillart [Salginatobel]. The main characteristic of this beautifully engineered artwork is that the extrados and the intrados of the reinforced concrete arch does in fact represent the envelope of the funicular curve - just in the way as explained above in the Fig. $2.4-$ b.c. 


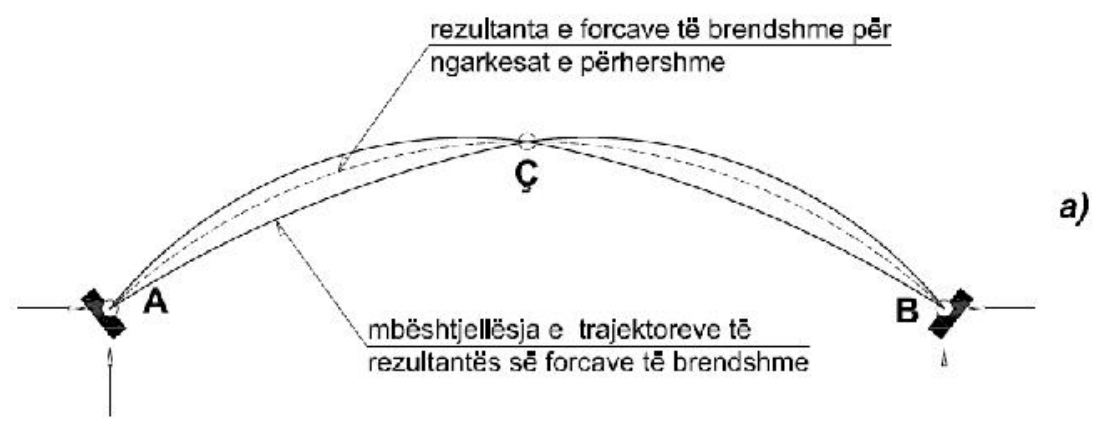

Fig. 2.4 a) Three-hinged arch - envelope of funicular curves; b) Sälginatobel bridge, Schiers, Graubünden/CH, 1930 [Robert Maillart found].

This scheme and the photo given in the Fig. 2.4 - $b$ [Robert Maillart found.], only stamp the hypothese raised further above: a structure (work of art) shall be able to fulfil aesthetical expectations only and only then when it has fulfilled basic criteria imposed by physical (Nature's) laws. This wonderfully engineered structure fulfils both of them: this is why it is adored both from the neophyte and the structural engineers. Capolavoro - in terms of Bruno Zevi [Capolavori del XX secolo]!

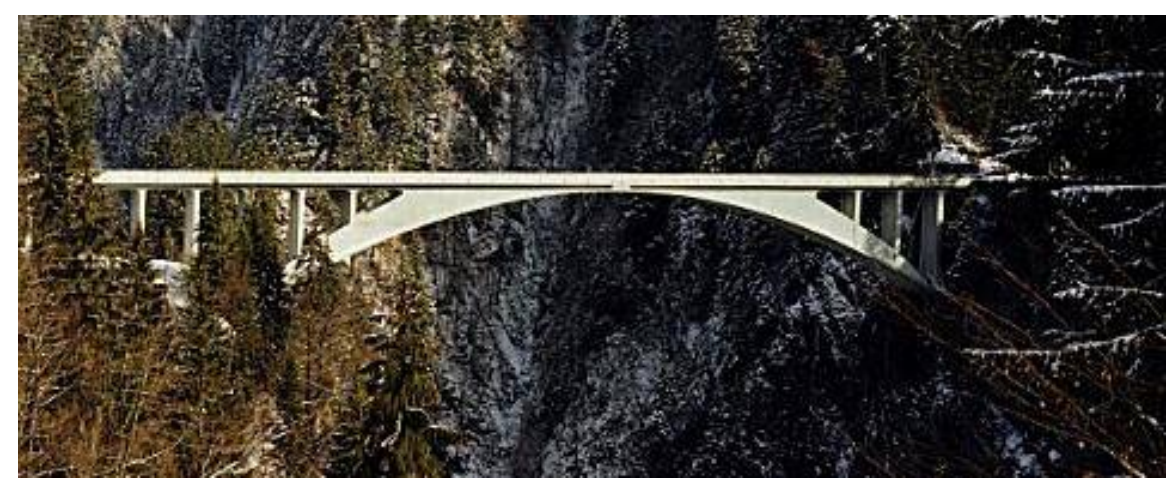

Fig. 2.5 Left: nomad's tent - cable net; middle: equilibrium of forces - child held by parents; right: primitive footbridge - structure working principally in tension 


\subsection{Traction (tension)}

The phenomena of traction (tension) lie at the very heart of dualism in nature and could be explained through Newton's Third Law or even (as given further above) by the universal gravitational law (expression 2.1). Naturally, this is valid only for the phenomena occurring outside the context of relativistic world, that is, for speeds much lower than the speed of light $(\sim 300000 \mathrm{~km} / \mathrm{s})$.

In the theory of structures this is of course valid.

One must stress that within a cross-section of a three-dimensional structural element - six internal effects of actions [Eurocode 0] appear. In the simplest of the cases (pure flexion) - two components results: traction and compression. Therefore, both tension and compression are incontournable to each other: where there is compression, there must be compression and vice versa.

An important fact here is: whilst structural elements submitted to compression requires sometimes requires a rather considerable amount of matter, those under the action of tension does require a very small amount of it. In some cases, as in the case of interplanetary attraction - there is no need for matter at all!

To turn back to the civil engineering structures, whilst bearing capacity of structural elements submitted to compression is constrained by their slenderness, the bearing capacity of structural elements submitted to the tension is borned by the mechanical resistance of their constitutive materials.

Now, the structural elements corresponding the best to the traction (tension) is the cable, which is one of the most used structural elements. Having into consideration its measures as well as the high degree of flexibility, there is only traction effort that can arise within it. This means the cable doesn't possess any flexional or torsion stiffness. Exempt from this is of course the prestressed cable or net of cables

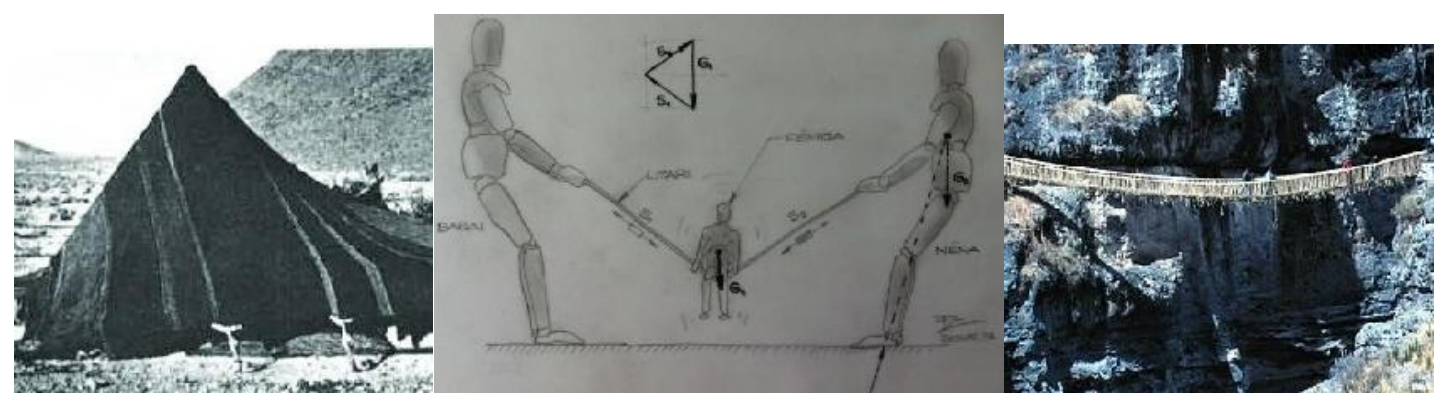

Fig. 2.6 below, shows three different structures, working principally in tension. On the left insert of the figure it is shown a nomads tent [Wikipedia] which represent nothing else than a set of threedimensional net of cables very near to each other with different curvatures in two orthogonal directions, whereas in the right insert one can see a primitive suspended bridge - made by nearby-found materials.

In the middle insert of Fig.2.6, one can see a sketch (made by the author of this paper), which does speak for itself, and illustrates the case of one of the most sophisticated biostructures in the universe (as such as we know) - the human body, together with the Cremona's polygon of forces shown as an insert within the figure in form of a triangle of force vectors in equilibrium. The external action is modelised by the child $G_{1}$ held by two parents $S_{1}$ and $S_{2}$.

Next in the Fig. 2.7 one can see the cable submitted to the action of a uniform load $g+p$, both for the symmetrical case and the antimetric one together with the deformed shapes the cable would take. 


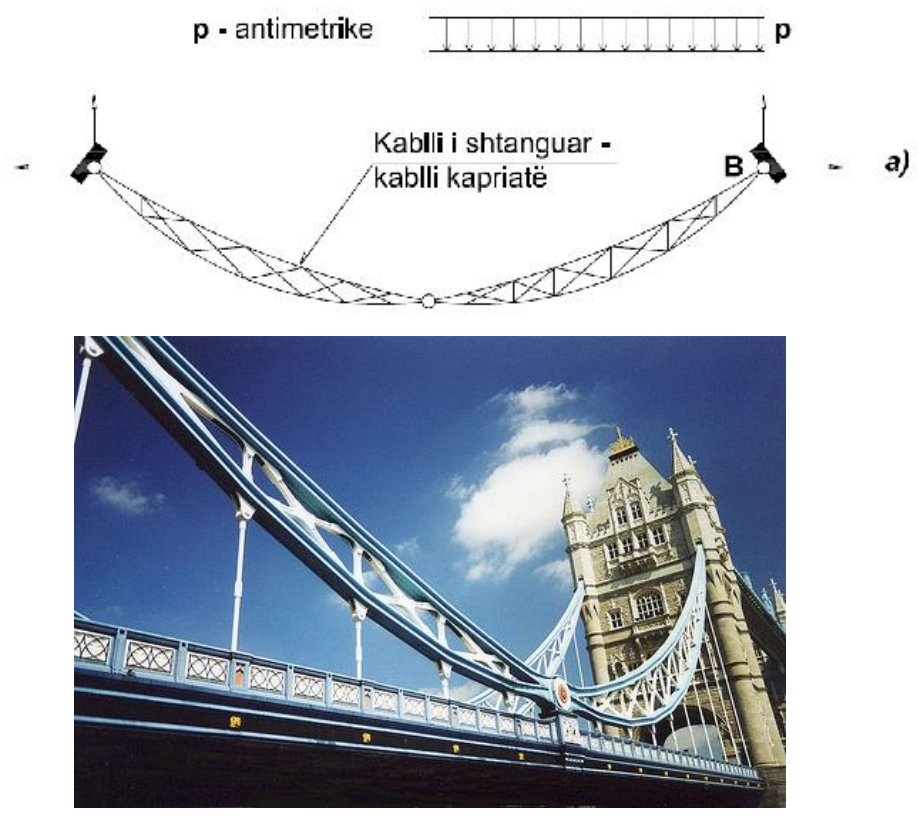

Fig. 2.7 Suspended cable over two supports at the same level: a) external actions $g+p$; b) variable antimetric action $p ; c)$ variable antimetric action $p$; continuous line - deformed shape, dashed line undeformed shape of the cable.

This short discourse will be closed by one of the most eminent cases in the history of the structural engineering - the "Tower Bridge" in London/UK. One of the most notable characteristics of this structure is the so called "fluxionally rigid" truss cable. The Fig. 2.8 below shows the extrados and the intrados of the cable -representing the envelope of the funicular polygon connected together by a system of vertical struts as well as diagonal ones.

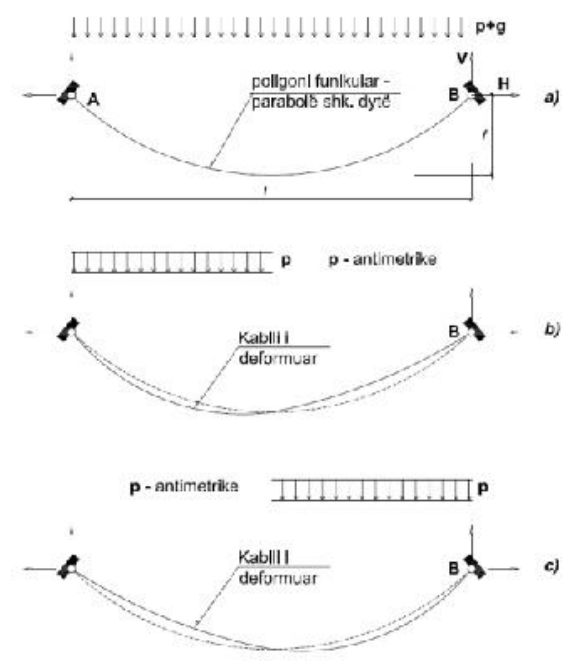

Fig. 2.8 a) Cantilevered beam; $b$ ) the mutual position of compression (dashed line) and traction (continuous line) for the case of dinosaurus; $c$ ) dinosaurus skleton [Wikipedia] - sketch shows only compression lines. 

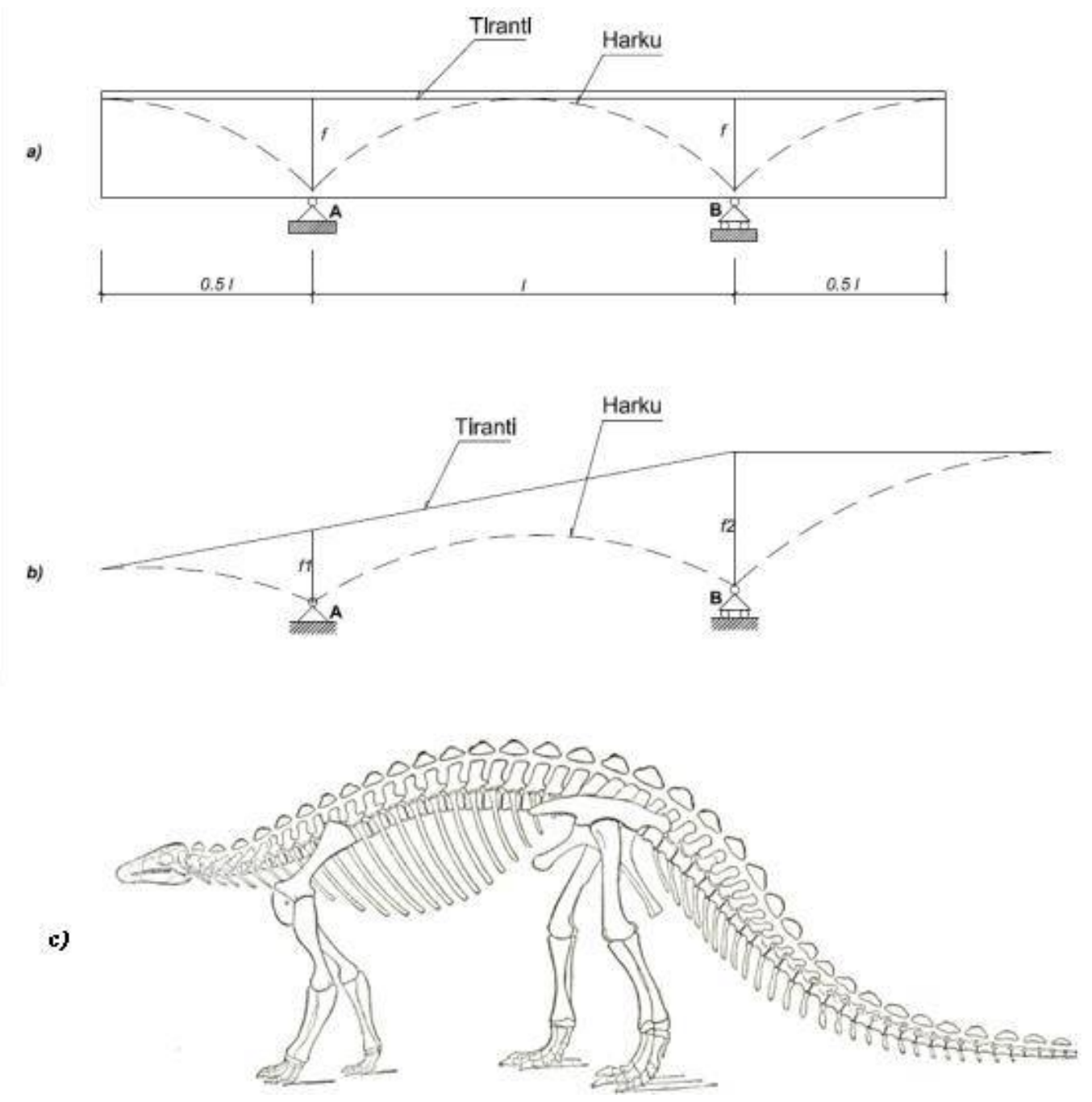

Fig. 2.9 Flexionally rigid - truss cable: a) the principle of stiffening and the analogy with Sälginatobel bridge; b) The "Tower Bridge" - London/UK [Ref].

\section{Compression and Traction - comparison with biostructures}

In the Fig. 2.8 hereunder, one can see the analogy between the cantilevered beam and the skeleton of dinosaurus. It is important to stress the similarity existing between the bending moment diagram of this very simple structure, and the shape of the skeleton. Here, one must stress the fact that only the components working under compression are visible (the bones) - since, organic parts representing the ligaments, muscles and tendons are gone long time ago.

\section{Conclusions}

In recent decades, we're witnessing major technological developments. In civil engineering in general, and in structural engineering particularly, powerful hardware and software makes us possible to achieve what have been unimaginable decades ago. Creating of 3D mathematical (analytical) models has become a routine, while super-fast processing and post-processing is done in a matter of minutes if not 
seconds. There is a tendency of putting too much belief in these results and in so doing; an inexperienced structural engineer can fast get into the trap of "beautiful" results given by the machine.

This paper represents a temptation to call for "awareness" within the community of structural designers (and not only), by the as I have named it:"return to the origin", or return to the basics on which our science of structures lays upon, that is the comprehension of the two principal genomes of the internal effects of actions in a cross-section of a structural element.

Nature gives us plenty of beautiful examples of how animals, plants ect., are growing and forms and shapes they are acquiring during this process - the biostructures. If we take a closer look at how these creatures are built, then, we could come to the conclusion that they are built by the same principles as are the civil engineering structures.

This paper, represents a modest contribution to this vast and unfortunately very little studied field which could become a source of great inspiration for structural engineering community.

\section{References}

1. Leonhardt, Fritz, Brücken: Ästhetik und Gestaltung = Bridges, Stuttgart, Deutsche VerlagsAnstalt, 1982.

2. Thompson, D'Arcy Wentworth, On growth and form, Cambridge University Press, Cambridge, UK, 1961.

3. Robert Maillart, Archives de la Société Suisse des Ingénieurs et des Architectes, Zürich.

4. EN 1990 Eurocode - Bazat e projektimit të Strukturave (engl. Basis of structural design) \& CEN TC $104-$ SC2.

5. Frey, François, Analyse des structures et milieux continus, Volume 1 jusq'à Volume 5, Presses Polytechniques et universitaires Romandes, 1990 à 2013.

6. Kurrer, Karl-Eugen, The History of the Theory of Structures-from arch analysis to computational mechanics, Ernst \& Sohn Verlag für Architektur und technische Wissenschaften GmbH \&Co. KG, 2008, Berlin.

7. Fuller, R., Bucminster, A. Loeb, E., J., Applewhite, Synergetics: Explorations in the Geometry of Thinking, Macmillan Pub Co, 1982.

8. F., H., Selmani, Anatomy of structures, Proceedings of the Second International Conference on Structures and Architecture, Guimarães, Portugal, 24-26 July 2013.

9. Selmani, F., Stavileci, M., Pjani, N., Mbi proporcionet dhe format racionale të strukturave inxhinierike, FORUM A+P, Universiteti POLIS, artikulli i pranuar për botim, 2013.

10. S., Timoshenko, Résistance des Matériaux $1^{\text {re }}$ Partie - Théorie élémentaire et problèmes, Paris et Liège, Librairie Polytechnique ch. Béranger, 1953.

11. Darwin, Ch., On the Origin of Species, Great Britain, 1998.

12. Peter Marti, Theory of Structures - Fundamentals, framed structures, plate shells, Ernst \& Sohn Verlag für Architektur und technische Wissenschaften GmbH \&Co. KG, 2013, Berlin. 